\title{
Non-invasive Estimation of Pressure Gradients in Pulsatile Flow using Ultrasound
}

Olesen, Jacob Bjerring; Villagómez Hoyos, Carlos Armando; Traberg, Marie Sand; Jensen, Jørgen Arendt

Published in:

Proceedings of IEEE International Ultrasonics Symposium

Publication date:

2014

Document Version

Early version, also known as pre-print

Link back to DTU Orbit

Citation (APA):

Olesen, J. B., Villagómez Hoyos, C. A., Traberg, M. S., \& Jensen, J. A. (2014). Non-invasive Estimation of Pressure Gradients in Pulsatile Flow using Ultrasound. In Proceedings of IEEE International Ultrasonics Symposium (pp. 2257-2260). IEEE.

\section{General rights}

Copyright and moral rights for the publications made accessible in the public portal are retained by the authors and/or other copyright owners and it is a condition of accessing publications that users recognise and abide by the legal requirements associated with these rights.

- Users may download and print one copy of any publication from the public portal for the purpose of private study or research.

- You may not further distribute the material or use it for any profit-making activity or commercial gain

- You may freely distribute the URL identifying the publication in the public portal 
Paper presented at the IEEE International Ultrasonics Symposium, Chicago, Il., USA, 2014:

\section{Non-invasive Estimation of Pressure Gradients in Pulsatile Flow using Ultrasound}

Jacob Bjerring Olesen, Carlos Armando Villagómez Hoyos, Marie Sand Traberg and Jørgen Arendt Jensen

Center for Fast Ultrasound Imaging,

Biomedical Engineering group, Department of Electrical Engineering, Bldg. 349,

Technical University of Denmark, DK-2800 Kgs. Lyngby, Denmark

To be published in Proceedings of IEEE International Ultrasonics Symposium, Chicago, Il., USA, 2014. 


\title{
Non-invasive Estimation of Pressure Gradients in Pulsatile Flow using Ultrasound
}

\author{
Jacob Bjerring Olesen, Carlos Armando Villagómez Hoyos, Marie Sand Traberg and Jørgen Arendt Jensen \\ Center for Fast Ultrasound Imaging, Dept. of Elec. Eng., Bldg. 349, \\ Technical University of Denmark, DK-2800 Kgs. Lyngby, Denmark
}

\begin{abstract}
This paper investigates how pressure gradients in a pulsatile flow environment can be measured non-invasively using ultrasound. The presented set-up is based on vector velocity fields measured on a blood mimicking fluid moving at a peak flow rate of $1 \mathrm{ml} / \mathrm{s}$ through a constricted vessel. Fields of pressure gradients are calculated using the Navier-Stokes equations. Flow data are acquired to a depth of $3 \mathrm{~cm}$ using directional synthetic aperture flow imaging on a linear array transducer producing 1500 image frames of velocity estimates per second. Scans of a carotid bifurcation phantom with a $70 \%$ constriction are performed using an experimental scanner. The performance of the presented estimator is evaluated by comparing its results to a numerical simulation model, which geometry is reconstructed from MRI data. The study showed pressure gradients varying from $0 \mathrm{kPa} / \mathrm{m}$ to $4.5 \mathrm{kPa} / \mathrm{m}$ with a maximum bias and standard deviation of $10 \%$ and $13 \%$, respectively, relative to peak estimated gradient. The paper concludes that maps of pressure gradients can be measured non-invasively using ultrasound with a precision of more than $85 \%$.
\end{abstract}

\section{INTRODUCTION}

Measuring the intra-vascular blood pressure and its changes across constricted regions helps as a diagnostic marker for several cardiovascular diseases. In the clinic today, such measures are mostly obtained by pressure catheters introduced to the femoral artery and threaded to the region of interest. Despite this procedure being widely accepted, it suffers some severe limitation on both the accuracy of the measurement, and that it is a highly invasive procedure requiring ionizing radiation for X-ray guidance. Recently, Adelaide et al. [1] published a study on how the physical size and shape of a pressure catheter affects the accuracy of the intended measurement. The study showed that a routinely employed catheter induces a $24 \%$ overestimation of the peak systolic pressure compared to a wire estimate, which is considered the golden standard [2].

An interest of replacing invasive catheters by less invasive methods started to grow in the 1970's. In 1976, Holen et al [3] introduced the first fully noninvasive alternative for estimating intra-vascular pressure differences. They suggested a method that estimated local pressure gradients based on the peak systolic blood velocity measured using Doppler ultrasound. This noninvasive estimate may have moved itself from the errors related to catheterization, but were now facing the difficulties associated with ultrasound scanners of that time, e.g. poor signal-to-noise ratio and bad temporal and spatial resolution. Most damaging to the method was its solely dependence on a single velocity estimate, which made the method highly sensitive to hemodynamic factors that were unrelated to the constricted vessel's effect on the peak velocity, e.g., abnormal cardiac output. The last decades has led to advancements in noninvasive techniques for improving pressure estimates [4][6], but none of them have successfully managed to substitute pressure catheters in the clinic. This paper presents an estimator that relies on vector velocity data acquired at 1500 frames per second with a precision of $98 \%$ to the true value [7]. The authors believe that a method, which operates at a frame rate capable of capturing every flow feature that exist throughout the cardiac cycle, and has a high precision, is the key to one day substituting or assisting catherization.

\section{DERIVING PRESSURE GRADIENTS}

The following section presents a method for calculating pressure gradients using ultrasound vector velocity data. The method is based on Navier-Stokes equations for an isotropic incompressible Newtonian fluid:

$$
\rho\left[\frac{\partial \vec{v}}{\partial t}+\vec{v} \cdot \nabla \vec{v}\right]=-\nabla p+\rho \vec{g}+\mu \nabla^{2} \vec{v}
$$

The equation describes the development of a fluid's velocity $\vec{v}\left(v_{x}, v_{y}, v_{z}\right)$ by relating the body forces acting on the isotropic volume to its acceleration and density, where $\rho$ is the density of the fluid and $\mu$ its viscosity. The left-hand side sums the local $\frac{\partial \vec{v}}{\partial t}$ and convective fluid acceleration $\vec{v} \cdot \nabla \vec{v}$, where $\nabla$ is the spatial differential operator $\left(\frac{\partial}{\partial x}, \frac{\partial}{\partial y}, \frac{\partial}{\partial z}\right)$, while the right-hand side sums the surface and volume forces. The terms on the right-hand side include the pressure gradients $-\nabla p$, as well as a gravitational force $\mathbf{g}$ and viscous drag caused by the viscosity of the fluid $\mu \nabla^{2} \vec{v}$, where $\nabla^{2} \vec{v}$ is the Laplacian of the velocity. The gravitational term can usually be neglected, as a patient undergoing an ultrasound scan is placed in a supine position.

Eq. (1) states that all three vector components of $\vec{v}\left(v_{x}, v_{y}, v_{z}\right)$ must be known to estimate the pressure gradient $\nabla p$. However, the method employed in this study image only the twodimensional (2-D) in-plane velocity vector $\vec{v}=\left(v_{x}, v_{z}\right)$. The proposed method is therefore developed assuming that the outof-plane velocity $v_{y}$ is zero. The reduced form of the NavierStokes equations are expressed here in rectangular coordinates,

$$
\left[\begin{array}{l}
\frac{\partial p}{\partial x} \\
\frac{\partial p}{\partial z}
\end{array}\right]=-\rho\left[\begin{array}{l}
\frac{\partial v_{x}}{\partial t}+v_{x} \frac{\partial v_{x}}{\partial x}+v_{z} \frac{\partial v_{x}}{\partial z} \\
\frac{\partial v_{z}}{\partial t}+v_{x} \frac{\partial v_{z}}{\partial x}+v_{z} \frac{\partial v_{z}}{\partial z}
\end{array}\right]
$$




$$
+\mu\left[\begin{array}{l}
\frac{\partial^{2} v_{x}}{\partial x^{2}}+\frac{\partial^{2} v_{x}}{\partial z^{2}} \\
\frac{\partial^{2} v_{z}}{\partial x^{2}}+\frac{\partial^{2} v_{z}}{\partial z^{2}}
\end{array}\right] .
$$

The derivatives in (2) are calculated from velocity data using polynomial filtering. A second-order polynomial is fitted to a subset of adjacent data points by the linear least-squared method, which results in a smoothed data set. From this data set the first and second-order derivatives are found using the convolution coefficients [8], [9].

\section{DATA ACQUISITION AND EXPERIMENTAL SET-UP}

Velocity data are acquired on a flow phantom mimicking the carotid bifurcation having a $70 \%$ constriction of the internal branch, (C70-SSEA Shelley Medical Imaging Technologies, Toronto, Canada). Ultrasound data are recorded over the constriction using a BK8670 linear array transducer connected to the experimental research scanner SARUS [10], capable of sampling individual RF data from 1024 channels. The flow estimator uses synthetic aperture imaging together with focusing in receive along the direction of the flow as suggested by Jensen and Nikolov [7]. The obtained signals are then crosscorrelated to find the magnitude of the velocity. The carotid bifurcation phantom is connected to a flow system (CompuFlow 1000, Shelley Medical Imaging Technologies, Toronto, Canada) capable of generating time-varying waveforms. The pump is set to produce a waveform that mimics that of a carotid artery, see Fig. 1.

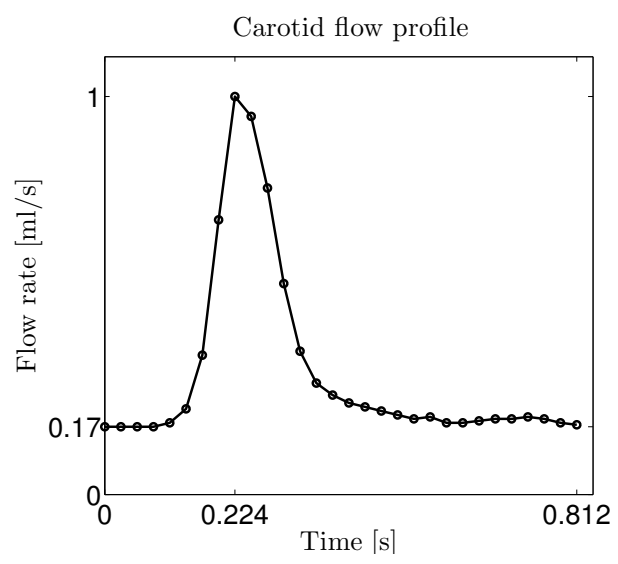

Fig. 1. Waveform produced by the pulsating flow pump

Data are recorded over three full cycles, producing roughly 3700 frames of velocity. Each velocity frame initially contains of $120 \times 11$ estimates, spanning an area of $11 \times 10 \mathrm{~mm}$. The velocity data is then up-sampled by a factor of five in the lateral direction. Up-sampling is made to reduce the effects of wall-signal being smeared into the lumen of the vessel when calculating the derivatives of the selected subset of data points. A sliding window covering 225 frames $(\approx 0.15 \mathrm{~s})$ is used for smooothing and calculating the temporal acceleration from the estimated velocities data, while the spatial derivatives were found using window sizes of $31(\approx 3 \mathrm{~mm})$ and $11(\approx 2 \mathrm{~mm})$ data points for the axial and lateral direction, respectively.

The accuracy of the estimated pressure gradients is evaluated through comparison to a finite-element model produced in Comsol (Comsol v4.4, Comsol AB, Stockholm, Sweden). The geometry of this model is constructed from MRI data of the flow phantom obtained using a 3-T scanner (Magnetom Trio, Siemens AG, Munich, Germany) at the Department of Diagnostic Radiology at Rigshospitalet, Denmark. The flow parameters in the simulation model are set to mimic the actual flow conditions in the experimental set-up. An inlet profile equivalent to the one in Fig. 1 is set for the simulation model and the viscosity and density is set to match the properties of the blood-mimicking fluid (BMF-US, Shelley Medical Imaging Technologies, Toronto, Canada) used in the experimental setup.

\section{RESUlTS}

A map of vector velocities during peak systole is simulated and plotted for the center of the constricted vessel in Fig. 2A. The color indicates the magnitude of the velocity while the arrows point in the direction of flow movement. The flow accelerates toward the center of the constriction, reaching a peak velocity of $0.13 \mathrm{~m} / \mathrm{s}$, before decelerating as the crosssectional area expands again. Figs. $2 \mathrm{~B}$ and $2 \mathrm{C}$ show maps of pressure gradients derived from the velocity data plotted in Fig. 2A. Fig. 2B presents gradients that are the direct output from the simulation software Comsol, while Fig. 2C shows gradients derived from the simulated velocity data using the model suggested in (2). A comparison between the output from Comsol and the output from the model is made for the maximum pressure gradient found upstream to the constriction, indicated by the circle in the bright green region. The comparison is made for the axial and lateral gradient component and is plotted in Figs. 3-4. The root-mean-square deviation is calculated with respect to the observed range and is found to be $5.6 \%$ for the axial component and $4.5 \%$ for the lateral component. The resemblance between the Comsol output and the output from the suggested model indicates that the model is suitable for calculating pressure gradients.

The experimental results are plotted in Fig. 5. Fig. 5A shows vector velocities at peak systole estimated using the directional synthetic aperture flow imaging approach. As in the case with the simulated data, the flow accelerates toward the constriction reaching a peak velocity of approximately $0.15 \mathrm{~m} / \mathrm{s}$. The plotted velocities are used for deriving the pressure gradients. The result of this is seen in Fig. 5B. The overall appearance of the derived gradients shows the same tendencies as in the simulated set-up. Both cases display gradients that point away from the center of the constriction indicating that a low pressure is present here. The estimates at the vessel's boundaries are affected by the backscattering signal from the vessel wall, especially at the center of the constriction. This region is particular critical as high velocities are seen close to a region outside the flow domain, thus, producing large spatial decelerations, which creates false magnitudes in the derived pressure gradients. A 


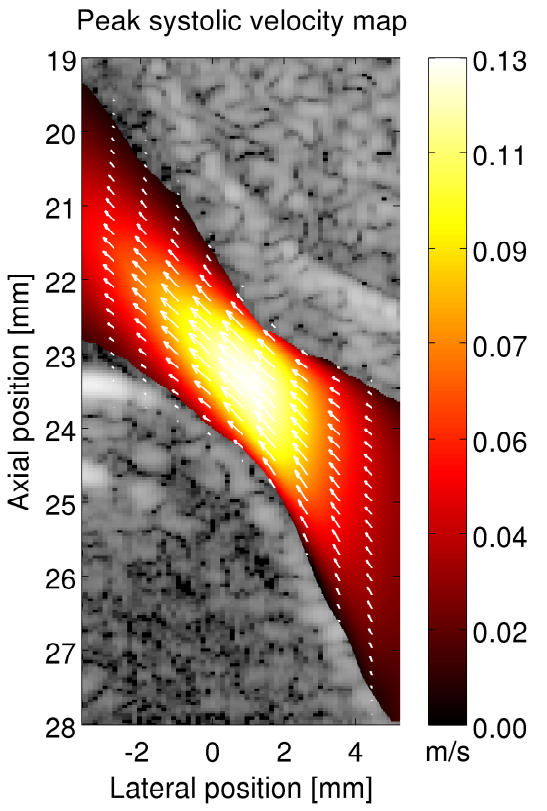

(a)

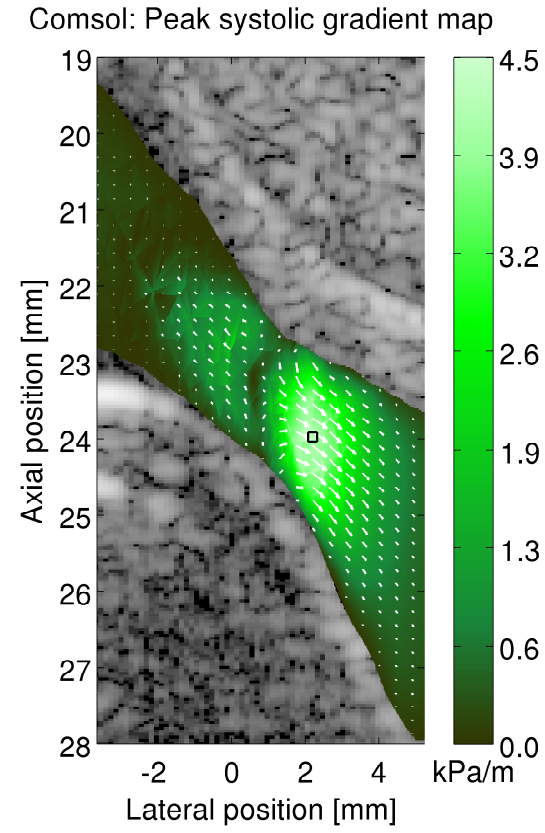

(b)

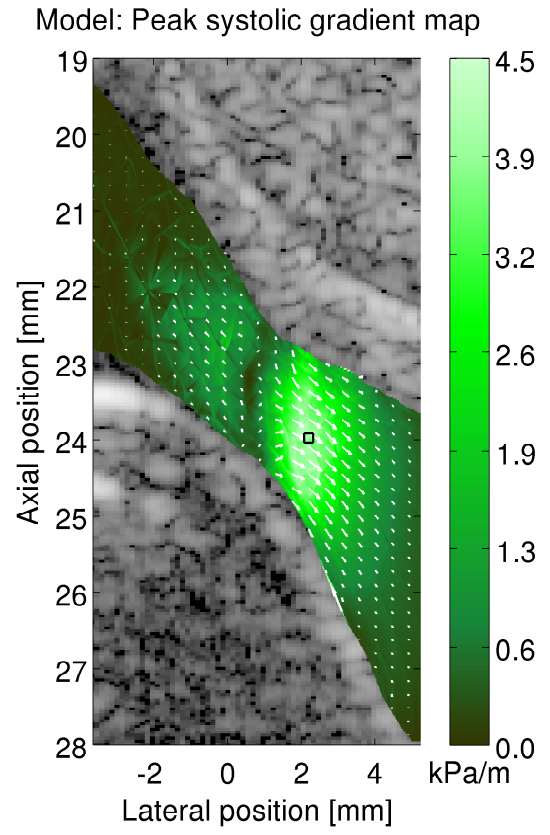

(c)

Fig. 2. Simulated data. (a) shows the simulated vector velocity field through the constricted region. (b) shows a map of the simulated pressure gradients. (c) display pressure gradients derived using (2) and the velocity data shown in (a).

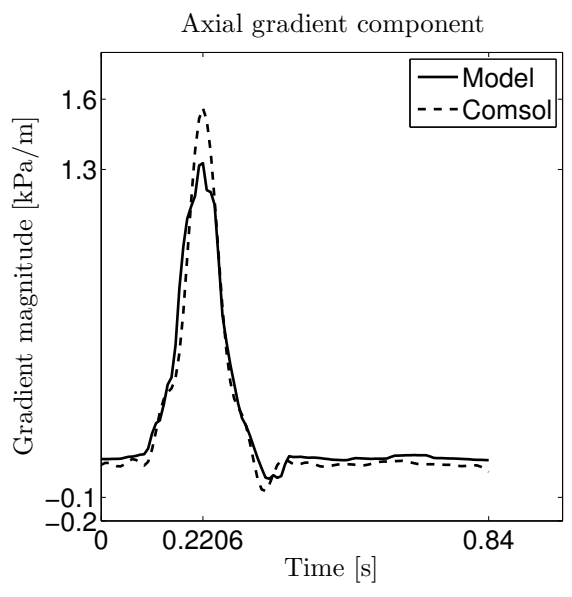

Fig. 3. Magnitude of axial pressure gradient component

comparison between the estimated results and the simulation model is made for the peak gradient in the upstream region, see Figs. 6 and 7. The maximum bias was found for the axial component, which reached $10 \%$ of the peak estimated gradient. The standard deviation over the three cycles was calculated to $13 \%$ for both the axial and lateral component.

\section{CONCLUSIONS}

A non-invasive method for deriving pressure gradients in a pulsatile flow set-up using vector velocity ultrasound data was presented. The pressure gradients were derived using the Navier-Stokes equations for incompressible fluids. The vector velocities inserted into the equations were estimated

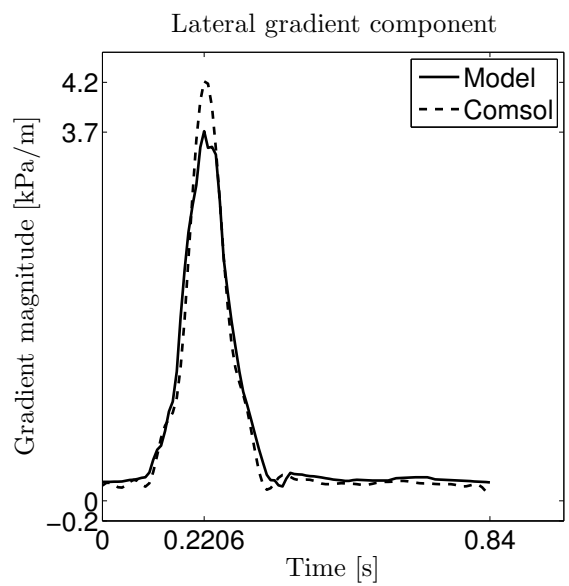

Fig. 4. Magnitude of lateral pressure gradient component

using the directional synthetic aperture flow imaging at 1500 frames per second. Measurements were performed on a constricted carotid phantom and the results were compared to a numerical simulation model. The experiment showed at maximum bias of $10 \%$ and a relative standard deviation of $13 \%$. The paper concludes that pressure gradients can be measured non-invasively using ultrasound to a precision of $85 \%$ when looking over three cardiac cycles.

\section{REFERENCES}

[1] A. de Vecchi, R. E. Clough, N. R. Gaddum, M. C. M. Rutten, P. Lamata, T. Schaeffter, D. A. Nordsletten, and N. P. Smith, "Catheter-induced 


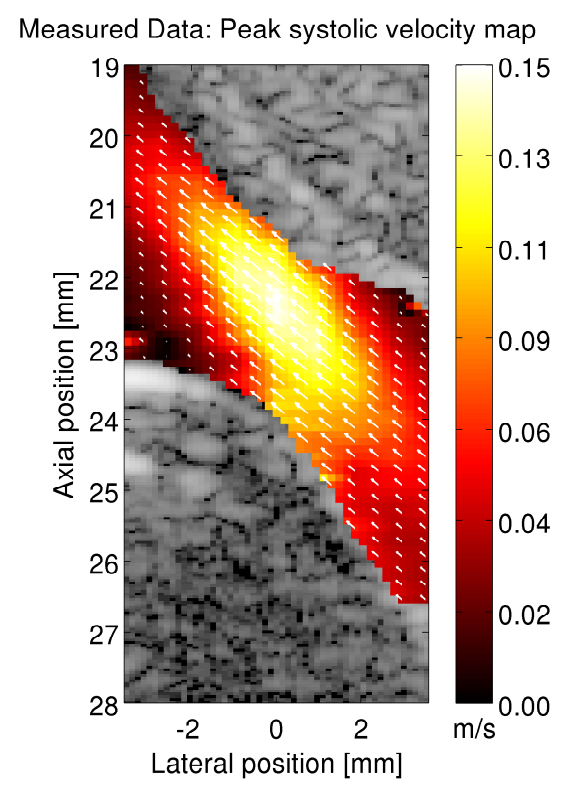

(a)

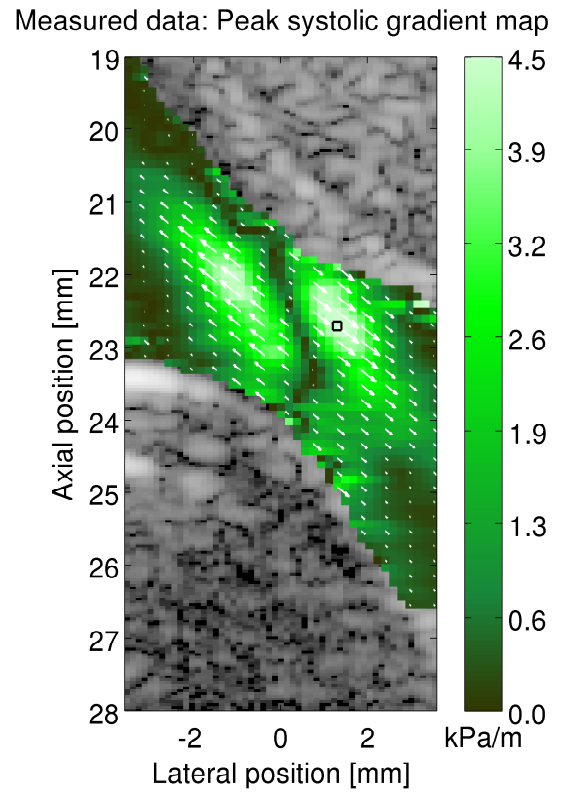

(b)

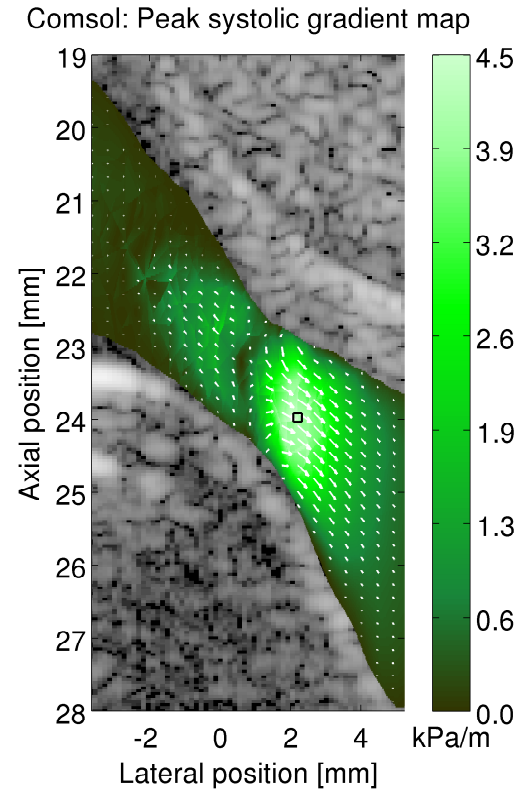

(c)

Fig. 5. Measured data. (a) shows the estimated vector velocity field through the constricted vessel. (b) displays a map of pressure gradients, estimated from the velocity data in (a) using (2). (c) shows the simulated pressure gradients, (same plot as Fig. 2b)

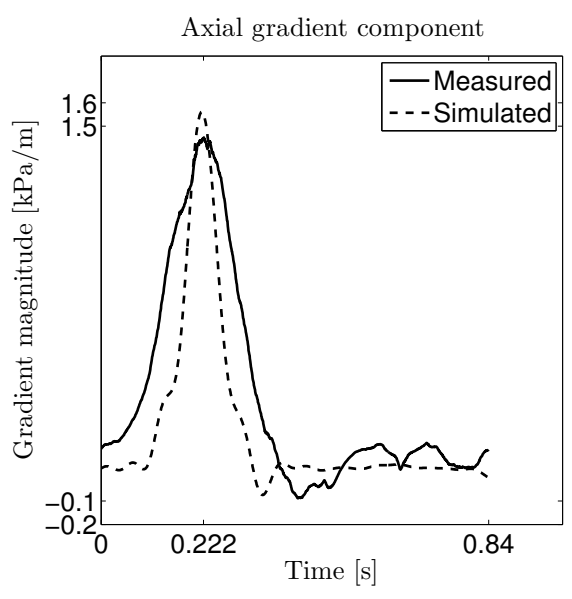

Fig. 6. Magnitude of axial pressure gradient component

errors in pressure measurements in vessels: An in-vitro and numerical study," IEEE Trans. Biomed. Eng., vol. 61, no. 6, pp. 1844-1850, 2014.

[2] J. J. Cavendish, L. I. Carter, and S. Tsimikas, "Recent advances in hemodynamics: Noncoronary applications of a pressure sensor angioplasty guidewire," Catheter Cardiovasc Interv, vol. 71, no. 6, pp. 748-758, 2008.

[3] J. Holen, R. Aaslid, and K. Landmark, "Determination of pressure gradient in mitral stenosis with a non-invasive ultrasound Doppler technique," Acta med. scand., vol. 32, pp. 455-460, 1976.

[4] S. Ohtsuki and M. Tanaka, "Doppler pressure field deduced from the Doppler velocity field in an observation plane in a fluid," Ultrasound Med. Biol., vol. 29, no. 10, pp. 1431-1438, 2003.

[5] A. Herment, G. Besson, C. Pellot-Barakat, and F. Frouin, "Estimation of pressure gradient images from velocity encoded MR acquisitions," Computers in Cardiology, vol. 35, pp. 933-936, 2008.

[6] J. P. Tasu, E. Mousseaux, A. Delouche, C. Oddou, O. Jolivet, and
J. Bittoun, "Estimation of pressure gradients in pulsatile flow from Lateral gradient component

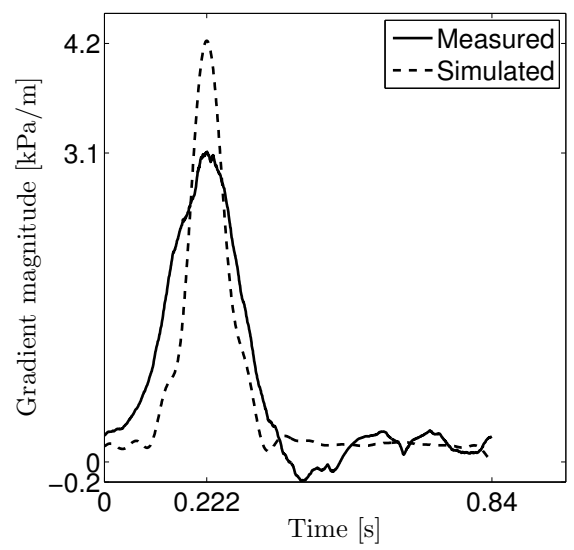

Fig. 7. Magnitude of lateral pressure gradient component

magnetic resonance acceleration measurements," Magn. Reson. Med., vol. 44, no. 1, pp. 66-72, 2000.

[7] J. A. Jensen and S. I. Nikolov, "Directional synthetic aperture flow imaging," IEEE Trans. Ultrason., Ferroelec., Freq. Contr., vol. 51, pp. 1107-1118, 2004.

[8] A. Savitzky and M. J. E. Golay, "Smoothing and differentiation of data by simplified least squares procedures," Anal. Chem., vol. 36, no. 8, pp. 1627-1639, 1964.

[9] J. B. Olesen, M. S. Traberg, M. J. Pihl, and J. A. Jensen, "Noninvasive esimation of 2-D pressure gradients in steady flow using ultrasound," IEEE Trans. Ultrason., Ferroelec., Freq. Contr., vol. 61, no. 8, pp. 1409 $1418,2014$.

[10] J. A. Jensen, H. Holten-Lund, R. T. Nilsson, M. Hansen, U. D. Larsen, R. P. Domsten, B. G. Tomov, M. B. Stuart, S. I. Nikolov, M. J. Pihl, Y. Du, J. H. Rasmussen, and M. F. Rasmussen, "SARUS: A synthetic aperture real-time ultrasound system," IEEE Trans. Ultrason., Ferroelec., Freq. Contr., vol. 60, no. 9, pp. 1838-1852, 2013. 